DOI: $10.2478 / \mathrm{v} 10324-012-0016-1$<smiles>C1=C2C=C(C1)C2</smiles>

VERSITA
Analele Universităţii de Vest, Timişoara

Seria Matematică - Informatică L, 2, (2012), 67-83

\title{
On Radial Continuity of Metric Projections
}

T. D. Narang and Shavetambry Tejpal

\begin{abstract}
Some new continuity concepts called Outer Radially Lower(ORL), Outer Radially Upper (ORU) and Inner Radially Lower (IRL), for set-valued metric projections were introduced in Banach spaces by B. Brosowski and F. Deutsch [Bull. Amer. Math. Soc. 78(1972), 974-978] to characterize suns and Chebyshev sets. In this paper we extend these concepts together with the concept of Inner Radially Upper (IRU) continuity to convex metric spaces and prove some results including those of Brosowski and Deutsch in such spaces.
\end{abstract}

AMS Subject Classification (2000). 41A65, 54C60.

Keywords. Radial continuity, metric projection, Chebyshev set, sun, moon, boundedly connected set, convex metric space, Mspace.

\section{Introduction}

Let $V$ be a non-empty subset of a metric space $(X, d)$. The metric projection onto $V$ is the mapping $P_{V}$, which takes each element $x$ of 
$X$ into the set of (global) best approximations to $x$ in $V$ i.e. $P_{V}(x)=$ $\{v \in V: d(x, v)=\operatorname{dist}(x, V)\} . V$ is called proximinal if $P_{V}(x) \neq \emptyset$ for each $x \in X$ and Chebyshev if $P_{V}(x)$ contains exactly one point for each $x \in X . P_{V}$ is called lower semi-continuous (upper semi-continuous) at $x$ if for each open set $W$ with $P_{V}(x) \cap W \neq \emptyset\left(P_{V}(x) \subset W\right)$ there exists a neighbourhood $U$ of $x$ such that $P_{V}(y) \cap W \neq \emptyset\left(P_{V}(y) \subset W\right)$ for all $y \in U$. Upper semi-continuity and lower semi-continuity of the metric projections have played a key role in discussing the structure of the set $V$ and the geometry of the space (see [4] and references cited therein). Brosowski and Deutsch [3], [4] introduced in normed linear spaces some simple and more general 'radial' continuity criteria (called Outer Radial Lower, Inner Radial Lower and Outer Radial Upper), which require that the restriction of the metric projection to certain prescribed line segments be lower semi continuous (lsc) or upper semi continuous (usc). It turned out (see [3], [4]) that these criteria, which are much weaker than lsc or usc, were strong enough to generalize a number of known results and weak enough so that many of the known theorems have valid converses (which they did not have under the strong hypotheses of lsc or usc). In this paper we extend these concepts and also the concept of Inner Radially Upper (IRU) continuity introduced in normed linear spaces by Panda [10] to convex metric spaces and prove some results including those of Brosowski and Deutsch in such spaces. We shall see that for suns, the metric projection is ORL-continuous, for Chebyshev sets the metric projection is IRL and IRU-continuous and for closed convex set whose metric projection is compact-valued, the metric projection is ORU-continuous. A set having both IRL and ORU continuous metric projection is shown to be boundedly connected and have a connected-valued metric projection. The proofs of some of the lemmas and subsequent theorems given in this paper follow the line of arguments presented in [4] and [8] with suitable modifications.

\section{Notations and Definitions}

In this section we give some notations and recall few definitions. Let $(X, d)$ be a metric space and $x, y, z \in X$. We say that $z$ is between $x$ 
and $y$ if $d(x, z)+d(z, y)=d(x, y)$. For any two points $x, y$ of $X$, the set $\{z \in X: d(x, z)+d(z, y)=d(x, y)\}$ is called the metric segment and is denoted by $G[x, y]$. The set $G[x, y,-]$ denotes the ray starting from $x$ and passing through $y$. The set $G_{1}(x, y,-) \equiv G[x, y,-] \backslash G[x, y]$ i.e. it is the set of all those points on the ray starting from $x$ and passing through $y$ which do not lie between $x$ and $y$.

A continuous mapping $W: X \times X \times[0,1] \rightarrow X$ is said to be a convex structure on $X$ if for all $x, y \in X$ and $\lambda \in[0,1]$

$$
d(u, W(x, y, \lambda)) \leqslant \lambda d(u, x)+(1-\lambda) d(u, y)
$$

holds for all $u \in X$. A metric space $(X, d)$ together with a convex structure is called a convex metric space [12].

A convex metric space $(X, d)$ is said to satisfy $\operatorname{Property}(I)[6]$ if for all $x, y \in X$ and $\lambda \in[0,1]$,

$$
d(W(x, p, \lambda), W(y, p, \lambda)) \leqslant \lambda d(x, y)
$$

where $p$ is arbitrary but fixed point of $X$.

A convex metric space $(X, d)$ is called strongly convex [11] or an $M$ space [7] if for every two points $x, y \in X$ and every $r \in[0,1]$ there exists unique point $z_{r}=W(x, y, r) \in X$ such that $d\left(x, z_{r}\right)=(1-r) d(x, y)$ and $d\left(y, z_{r}\right)=r d(x, y)$ i.e. unique point $z_{r}$ of the metric segment $G[x, y]$. A metric space $(X, d)$ is called externally convex [7] if for all distinct points $x, y$ such that $d(x, y)=\lambda$, and $r>\lambda$ there exists a unique $z$ of $X$ such that $d(x, y)+d(y, z)=d(x, z)=r$.

A subset $K$ of a convex metric space $(X, d)$ is said to be convex [12] if $W(x, y, \lambda) \in K$ for all $x, y \in K$ and $\lambda \in[0,1]$ i.e. $G[x, y] \subset K$ for all $x, y \in K$.

If $V$ is a proximinal subset of an externally convex M-space $(X, d)$, a point $v_{0} \in V$ is called a solar point of $V$ if $v_{0} \in P_{V}(x)$ implies $v_{0} \in P_{V}\left(x_{\lambda}\right)$ for every $x_{\lambda} \in G_{1}\left(v_{0}, x,-\right)$. The set $V$ is called a sun [5] if for each $x \in X \backslash V$, every $v_{0} \in P_{V}(x)$ is a solar point of $V$. A point $v_{0} \in V$ is called a lunar point if $v_{0} \in P_{V}(x)$ and $K\left(v_{0}, x\right) \cap V \neq \emptyset$ imply $v_{0} \in \overline{\left.K\left(v_{0}, x\right) \cap V\right)}$ where $K\left(v_{0}, x\right) \equiv \bigcup_{z} B\left(z, d\left(z, v_{0}\right)\right), z \in G_{1}\left(v_{0}, x,-\right)$ and $B(z, r)$ stands for open ball with centre $z$ and radius $r$. The set $V$ is called a moon [1] if each of its point is lunar.

A point $v_{0} \in V$ is called a local best approximation (LBA) to $x$ in $V$ if there is an $r>0$ such that $v_{0} \in P_{V \cap B\left(v_{0}, r\right)}(x)$. Clearly each best 
approximation to $x$ in $V$ is a LBA but not conversely (see e.g. Braess $[2]$, p.38).

\section{Outer Radial Lower (ORL) Continuity}

First generalization of lower semi continuity (lsc) is the following: Let $V$ be a non-empty subset of an externally convex M-space $(X, d)$ and $x_{0} \in X$. We say that $P_{V}$ is ORL-continuous at $x_{0}$ if for each $v_{0} \in P_{V}\left(x_{0}\right)$ and each open set $W$ for which $P_{V}\left(x_{0}\right) \cap W \neq \emptyset$ there exists a neighbourhood $U$ of $x_{0}$ such that $P_{V}(x) \cap W \neq \emptyset$ for all $x \in$ $U \cap G_{1}\left(v_{0}, x_{0},-\right) . P_{V}$ is called ORL-continuous if it is ORL-continuous at each point.

The following lemma, which was proved for normed linear spaces by Brosowski and Deutsch ([4] - Lemma 2.2) and holds for externally convex M-spaces will be used in proving our first theorem:

Lemma 3.1. Let $V$ be a non-empty subset of an externally convex $M$-space $(X, d)$ and $x_{0} \in X$ then the following are equivalent:

1. $P_{V}$ is ORL-continuous at $x_{0}$,

2. for each $v_{0}, v_{1} \in P_{V}\left(x_{0}\right)$ and each $\epsilon>0$ there exists a $\delta>0$ such that $P_{V}(x) \cap B\left(v_{1}, \epsilon\right) \neq \emptyset$ for all $x \in\left\{z: d\left(z, x_{0}\right)<\delta\right.$ and $\left.z \in G_{1}\left(v_{0}, x_{0},-\right)\right\}$,

3. for each $v_{0}, v_{1} \in P_{V}\left(x_{0}\right)$ and every sequence $z_{n}$ in $G_{1}\left(v_{0}, x_{0},-\right)$ with $z_{n} \rightarrow x_{0}$ there exists $v_{n} \in P_{V}\left(z_{n}\right)$ such that $v_{n} \rightarrow v_{1}$.

Theorem 3.1. For a non-empty subset $V$ of an externally convex $M$ space $(X, d)$, consider the following:

1. $V$ is a sun,

2. The metric projection $P_{V}$ is ORL-continuous,

3. for each $x \in X$, every local best approximation is a global best approximation,

4. $V$ is a moon, 
then $(1) \Rightarrow(2) \Rightarrow(3) \Rightarrow(4)$.

Proof. $(1) \Rightarrow(2)$ Suppose $V$ is a sun. Let $x_{0} \in X, v_{0}, v_{1} \in P_{V}\left(x_{0}\right)$ and $\epsilon>0$ be given. In view of Lemma 3.1, it is sufficient to show that $P_{V}(z) \cap B\left(v_{1}, \epsilon\right) \neq \emptyset$ for all $z \in G_{1}\left(v_{0}, x_{0},-\right)$. As $V$ is a sun, $v_{0} \in P_{V}(z)$ for each $z \in G_{1}\left(v_{0}, x_{0},-\right)$ i.e. for each $z$ satisfying $d\left(z, v_{0}\right)=$ $d\left(z, x_{0}\right)+d\left(x_{0}, v_{0}\right)$. Consider

$$
\begin{aligned}
d\left(z, v_{1}\right) & \leqslant d\left(z, x_{0}\right)+d\left(x_{0}, v_{1}\right) \\
& =d\left(z, v_{0}\right)-d\left(x_{0}, v_{0}\right)+d\left(x_{0}, v_{1}\right) \\
& =d\left(z, v_{0}\right)-d\left(x_{0}, V\right)+d\left(x_{0}, V\right) \\
& =d\left(z, v_{0}\right) \\
& \leqslant d\left(z, v_{1}\right) \text { as } v_{0} \in P_{V}(z) .
\end{aligned}
$$

This gives $d\left(z, v_{0}\right)=d\left(z, v_{1}\right)$ and so $v_{1} \in P_{V}(z)$ and hence $P_{V}(z) \cap$ $B\left(v_{1}, \epsilon\right) \neq \emptyset$.

$(2) \Rightarrow(3)$ Let $v_{0}$ be a LBA to $x_{0} \in X$ i.e. $v_{0} \in P_{V \cap B\left(v_{0}, r\right)}\left(x_{0}\right)$ for some $r>0$. Suppose $v_{0}$ is not GBA to $x_{0}$. Therefore $d\left(x_{0}, v_{0}\right) \leqslant d\left(x_{0}, v\right)$ for all $v \in B\left(v_{0}, r\right) \cap V$ but $d\left(x_{0}, v_{0}\right)>d\left(x_{0}, V\right)$. Let $x_{1}$ be the last point on $G\left[v_{0}, x_{0}\right]$ which has $v_{0}$ as a GBA in $V$ (such a point clearly exists since $v_{0} \in P_{V}\left(v_{0}\right)$ and $v_{0} \notin P_{V}\left(x_{0}\right)$ but $v_{0}$ is a LBA to $\left.x_{0}\right)$. Thus $d(x, V)<d\left(x, v_{0}\right)$ for all $x \in G\left[x_{1}, x_{0}\right] \backslash\left\{x_{1}\right\}$. Now $P_{V}\left(x_{1}\right) \cap B\left(v_{0}, r\right) \neq$ $\emptyset$, and so by ORL continuity we can find a neighbourhood $B\left(x_{1}, \delta\right)$ such that $P_{V}(x) \cap B\left(v_{0}, r\right) \neq \emptyset$ for all $x \in B\left(x_{1}, \delta\right) \cap G_{1}\left(v_{0}, x_{1},-\right)$. If $x_{\lambda} \in B\left(x_{1}, \delta\right) \cap G_{1}\left(v_{0}, x_{1},-\right)$ and $v \in P_{V}\left(x_{\lambda}\right) \cap B\left(v_{0}, r\right)$ then

$$
d\left(x_{\lambda}, v\right)=d\left(x_{\lambda}, V\right)<d\left(x_{\lambda}, v_{0}\right) .
$$

Also $d\left(v, v_{0}\right)<r$. Consider

$$
d\left(x_{0}, v_{0}\right)=d\left(x_{0}, x_{\lambda}\right)+d\left(x_{\lambda}, v_{0}\right)>d\left(x_{0}, x_{\lambda}\right)+d\left(x_{\lambda}, v\right) \geqslant d\left(x_{0}, v\right) .
$$

Therefore $v_{0}$ is not a LBA to $x_{0}$, which is a contradiction. Hence $v_{0}$ is a GBA to $x_{0}$.

$(3) \Rightarrow(4)$ Suppose $V$ is not a moon. Then there exists $v_{0} \in V$ and $x \in P_{V}^{-1}\left(v_{0}\right)$ with $K\left(v_{0}, x\right) \cap V \neq \emptyset$ such that $v_{0} \notin \overline{K\left(v_{0}, x\right) \cap V}$ i.e. there exists $r>0$ such that

$$
B\left(v_{0}, r\right) \cap\left[K\left(v_{0}, x\right) \cap V\right]=\emptyset \text { i.e. } B\left(v_{0}, r\right) \cap K\left(v_{0}, x\right) \subseteq X \backslash V .
$$


Now, by the definition of $K\left(v_{0}, x\right)$, implies

$$
B\left(z, d\left(z, v_{0}\right)\right) \subseteq K\left(v_{0}, x\right) \text { for every } z \in G_{1}\left(v_{0}, x,-\right) \text {. So, (3.1) }
$$

$$
B\left(v_{0}, r\right) \cap B\left(z, d\left(z, v_{0}\right)\right) \subseteq X \backslash V \text { for every } z \in G_{1}\left(v_{0}, x,-\right)
$$

As $K\left(v_{0}, x\right) \cap V \neq \emptyset$, there exists $u \in K\left(v_{0}, x\right) \cap V$ and so $u \in$ $B\left(z, d\left(z, v_{0}\right)\right)$ for some $z \in G_{1}\left(v_{0}, x,-\right)$ i.e. $d(z, u)<d\left(z, v_{0}\right)$ and so $v_{0}$ can never be a GBA to $z$ in $V$.

Now we show that $v_{0}$ is a LBA to $z$ so chosen. Suppose $z$ has $v$ ' other than $v_{0}$ as a LBA in the r-neighbourhood of $v_{0}$ in $V$ i.e. $v / \in$ $P_{V \cap B\left(v_{0}, r\right)}(z)$ and so $d\left(z, v^{\prime}\right)<d\left(z, v_{0}\right)$ which implies $v^{\prime} \in B\left(z, d\left(z, v_{0}\right)\right)$. Also $v^{\prime} \in B\left(v_{0}, r\right)$ and so $v^{\prime} \in B\left(z, d\left(z, v_{0}\right)\right) \cap B\left(v_{0}, r\right)$. But then (3.2) gives $v^{\prime} \in X \backslash V$, a contradiction. Therefore $v_{0}$ is a LBA but not a GBA to $z$, contradicting the hypothesis. Hence $V$ is a moon.

Note 3.1. For normed linear spaces above theorem was given by Brosowski and Deutsch [4]- Theorem 2.3 (see also Mhaskar and Pai [8]- Theorem 7, p.468). In general, none of the implications in Theorem 3.1 is reversible (see [4]).

We call a space $X$ an $M S$-space [4] if every moon in $X$ is a sun. In such a space all the conditions of the above theorem are equivalent. In particular, we have

Corollary 3.1. Let $X$ be an MS-space and $V \subset X$. Then $V$ is a sun if and only if $P_{V}$ is ORL-continuous.

\section{Inner Radial Lower(IRL) Continuity}

A second generalization of lsc is:

Let $V$ be a non-empty subset of a convex metric space $(X, d)$ and $x_{0} \in$ $X$. We say that $P_{V}$ is $I R L$-continuous at $x_{0}$ if for each $v_{0} \in P_{V}\left(x_{0}\right)$ and each open set $W$ with $P_{V}\left(x_{0}\right) \cap W \neq \emptyset$, there exists a neighbourhood $U$ of $x_{0}$ such that $P_{V}(x) \cap W \neq \emptyset$ for every $x \in U \cap G\left[v_{0}, x_{0}\right] . P_{V}$ is called IRL-continuous if it is IRL-continuous at each point of $X$. 
Each lsc metric projection is IRL continuous but converse is not true (see [4]).

The following lemma, which was proved for normed linear spaces by Brosowski and Deutsch [4] and holds for convex metric spaces, will be used in our next theorem:

Lemma 4.1. Let $V$ be a non-empty subset of a convex metric space $(X, d)$ and $x_{0} \in X$ then the following are equivalent:

1. $P_{V}$ is IRL-continuous at $x_{0}$,

2. for each $v_{0}, v_{1} \in P_{V}\left(x_{0}\right)$, and each $\epsilon>0$ there exists $a \delta>0$ such that $P_{V}(x) \cap B\left(v_{1}, \epsilon\right) \neq \emptyset$ for all $x$ in $G\left[v_{0}, x_{0}\right]$ with $d\left(x, x_{0}\right)<\delta$,

3. for each $v_{0}, v_{1} \in P_{V}\left(x_{0}\right)$, and every sequence $x_{n}$ in $G\left[v_{0}, x_{0}\right]$ with $x_{n} \rightarrow x_{0}, d\left(v_{1}, P_{V}\left(x_{n}\right)\right) \rightarrow 0$ (i.e. there exists $v_{n} \in P_{V}\left(x_{n}\right)$ such that $\left.v_{n} \rightarrow v_{1}\right)$.

Theorem 4.1. Let $(X, d)$ be a convex metric space satisfying Property (I) and $x \in X$. If $P_{V}(x)$ is convex then $P_{V}$ is IRL-continuous at $x$.

Proof. If $P_{V}(x)=\emptyset$, the result is trivially true. Let $v_{0}, v_{1} \in P_{V}(x)$ and $x_{n} \in G\left[x, v_{0}\right]$ with $x_{n} \rightarrow x$. Thus $x_{n}=W\left(x, v_{0}, 1-\epsilon_{n}\right)$ where $0 \leqslant \epsilon_{n} \leqslant$ 1 and $\epsilon_{n} \rightarrow 0$. Also $d\left(x_{n}, x\right)=\epsilon_{n} d\left(v_{0}, x\right), d\left(x_{n}, v_{0}\right)=\left(1-\epsilon_{n}\right) d\left(x, v_{0}\right)$. Let $v_{n}=W\left(v_{1}, v_{0}, 1-\epsilon_{n}\right)$. Then $v_{n} \in P_{V}(x)$ as $P_{V}(x)$ is convex and $v_{n} \rightarrow v_{1}$. Also

$$
\begin{aligned}
d\left(x_{n}, v_{n}\right) & =d\left(W\left(x, v_{0}, 1-\epsilon_{n}\right), W\left(v_{1}, v_{0}, 1-\epsilon_{n}\right)\right) \\
& \leqslant\left(1-\epsilon_{n}\right) d\left(x, v_{1}\right) \\
& =\left(1-\epsilon_{n}\right) d\left(x, v_{0}\right) \\
& =d\left(x_{n}, v_{0}\right) \\
& =d\left(x_{n}, V\right)
\end{aligned}
$$

as $x_{n} \in G\left[x, v_{0}\right]$ and $v_{0} \in P_{V}(x)$ imply $v_{0} \in P_{V}\left(x_{n}\right)$ [13]. This gives $v_{n} \in$ $P_{V}\left(x_{n}\right)$ and hence $P_{V}$ is IRL-continuous at $x$ by the above lemma.

Remark 4.1. The converse of above theorem is not true (see [4]).

Remark 4.2. Since for convex sets $V, P_{V}(x)$ is convex [9] and for Chebyshev sets $V, P_{V}(x)$ being singleton is convex, we have 
Corollary 4.1. If $V$ is a convex or a Chebyshev set in a convex metric space $(X, d)$ satisfying Property $(\mathrm{I})$ then $P_{V}$ is IRL-continuous.

Neither of the sufficient conditions of the corollary are necessary (see $[4])$.

\section{Outer Radial Upper(ORU) Continuity}

A first generalization of upper semi continuity is:

Let $V$ be a non-empty subset of an externally convex M-space $(X, d)$ and $x_{0} \in X$. We say that $P_{V}$ is ORU-continuous at $x_{0}$ if for each $v_{0} \in$ $P_{V}\left(x_{0}\right)$ and each open set $W \supset P_{V}\left(x_{0}\right)$ there exists a neighbourhood $U$ of $x_{0}$ such that $P_{V}(x) \subset W$ for all $x \in U \cap G_{1}\left(v_{0}, x_{0},-\right)$. $P_{V}$ is called ORU-continuous if it is ORU-continuous at each point of $X$. The following lemma, which for normed linear spaces is given in [4]Lemma 4.2, and holds in externally convex M-spaces deals with ORUcontinuity.

Lemma 5.1. Let $V$ be a non-empty subset of an externally convex $M$-space $(X, d)$ and $x_{0} \in X$. Consider the following statements:

1. $P_{V}$ is ORU-continuous at $x_{0}$.

2. For each $v_{0} \in P_{V}\left(x_{0}\right)$ and each $\epsilon>0$ there exists $a \delta>0$ such that

$$
\sup _{v \in P_{V}(x)} d\left(v, P_{V}\left(x_{0}\right)\right)<\epsilon
$$

for every $x$ in $G_{1}\left(v_{0}, x_{0},-\right)$ with $d\left(x, x_{0}\right)<\delta$.

3. For each $v_{0} \in P_{V}\left(x_{0}\right)$ and each sequence $x_{n}$ in $G_{1}\left(v_{0}, x_{0},-\right)$ with $x_{n} \rightarrow x_{0}, \sup _{v \in P_{V}\left(x_{n}\right)} d\left(v, P_{V}\left(x_{0}\right)\right) \rightarrow 0$.

4. For each $v_{0} \in P_{V}\left(x_{0}\right)$ and each sequence $x_{n}$ in $G_{1}\left(v_{0}, x_{0},-\right)$ with $x_{n} \rightarrow x_{0}$ and each sequence $v_{n}$ with $v_{n} \in P_{V}\left(x_{n}\right), d\left(v_{n}, P_{V}\left(x_{0}\right)\right) \rightarrow$ 0 . 
5. For each $v_{0} \in P_{V}\left(x_{0}\right)$ and each sequence $x_{n}$ in $G_{1}\left(v_{0}, x_{0},-\right)$ with $x_{n} \rightarrow x_{0}$ and each sequence $v_{n}$ with $v_{n} \in P_{V}\left(x_{n}\right)$ and $v_{n} \rightarrow v$, $v \in \overline{P_{V}\left(x_{0}\right)}$.

Then $(1) \Rightarrow(2) \Rightarrow(3) \Rightarrow(4) \Rightarrow(5)$.

Note 5.1. If in the above lemma, $P_{V}\left(x_{0}\right)$ is compact then $(4) \Rightarrow(1)$ and if $V$ is compact then $(5) \Rightarrow(1)$.

Remark 5.1. In general, the implication $(4) \Rightarrow(1)$ and $(5) \Rightarrow(1)$ are false (see [4]).

The following theorem gives conditions under which metric projection is ORU continuous:

Theorem 5.1. If $V$ is a sun in an externally convex $M$-space $(X, d)$ such that $P_{V}(x)$ is compact for every $x \in X$ then $P_{V}$ is ORU-continuous.

Before proving this theorem, we establish a lemma.

Lemma 5.2. If $V$ is a sun in an externally convex $M$-space $(X, d)$, $v_{0} \in P_{V}\left(x_{0}\right)$ and $x_{n}$ is a sequence in $G_{1}\left(v_{0}, x_{0},-\right)$ with $x_{n} \rightarrow x_{0}$ then $P_{V}\left(x_{0}\right)=\cap_{n=1}^{\infty} P_{V}\left(x_{n}\right)$.

Proof. Let $v \in P_{V}\left(x_{0}\right)$. Then for each $n$,

$$
\begin{aligned}
d\left(x_{n}, v\right) & \leqslant d\left(x_{n}, x_{0}\right)+d\left(x_{0}, v\right) \\
& =d\left(x_{n}, x_{0}\right)+d\left(x_{0}, v_{0}\right) \\
& =d\left(x_{n}, v_{0}\right) \\
& =d\left(x_{n}, V\right)
\end{aligned}
$$

as $v_{0} \in P_{V}\left(x_{0}\right)$ and $V$ is a sun, so $v_{0} \in P_{V}\left(x_{n}\right)$ [13]. Then (5.1) implies $v \in P_{V}\left(x_{n}\right)$ for each $n$ and hence $P_{V}\left(x_{0}\right) \subset \cap_{n=1}^{\infty} P_{V}\left(x_{n}\right)$.

Conversely if $v \in \cap_{n=1}^{\infty} P_{V}\left(x_{n}\right)$ then $d\left(x_{n}, v\right)=d\left(x_{n}, V\right)$ for each $n$. Consider

$$
\begin{aligned}
d\left(x_{0}, v\right) & \leqslant d\left(x_{0}, x_{n}\right)+d\left(x_{n}, v\right) \\
& =d\left(x_{0}, x_{n}\right)+d\left(x_{n}, V\right) \\
& \rightarrow d\left(x_{0}, V\right) .
\end{aligned}
$$

Therefore $v \in P_{V}\left(x_{0}\right)$. 
Proof of Theorem 5.1. Let $x_{0} \in X$ and $v_{0} \in P_{V}\left(x_{0}\right)$. Suppose $x_{n}$ is a sequence in $G_{1}\left(v_{0}, x_{0},-\right)$ with $x_{n} \rightarrow x_{0}$. As $V$ is a sun, $v_{0} \in P_{V}\left(x_{n}\right)$ for every $n$. Let $W$ be an open set with $W \supset P_{V}\left(x_{0}\right)$. By Lemma 5.2, $P_{V}\left(x_{0}\right)=\cap_{n=1}^{\infty} P_{V}\left(x_{n}\right)$.

We claim that $P_{V}\left(x_{n}\right) \subset P_{V}\left(x_{n-1}\right)$. Let $v \in P_{V}\left(x_{n}\right)$. Then $d\left(x_{n}, v\right)=$ $d\left(x_{n}, V\right)$. Consider

$$
\begin{aligned}
d\left(x_{n-1}, v\right) & \leqslant d\left(x_{n-1}, x_{n}\right)+d\left(x_{n}, v\right) \\
& =d\left(x_{n-1}, x_{n}\right)+d\left(x_{n}, V\right) \text { as } v \in P_{V}\left(x_{n}\right) \\
& =d\left(x_{n-1}, x_{n}\right)+d\left(x_{n}, v_{0}\right) \\
& =d\left(x_{n-1}, v_{0}\right) \text { as } x_{n} \in G_{1}\left(v_{0}, x_{0},-\right) \text { for all } n \\
& =d\left(x_{n-1}, V\right) \text { as } v_{0} \in \cap_{n=1}^{\infty} P_{V}\left(x_{n}\right)
\end{aligned}
$$

This implies $v \in P_{V}\left(x_{n-1}\right)$ and $P_{V}\left(x_{n}\right) \subset P_{V}\left(x_{n-1}\right)$ for all $n$. Thus $P_{V}\left(x_{n}\right)$ is a decreasing sequence of compact sets. Let $v_{n} \in P_{V}\left(x_{n}\right), n=$ $1,2, \ldots$ Then $v_{n} \in P_{V}\left(x_{1}\right)$ for all $n$. Since $P_{V}\left(x_{1}\right)$ is compact, $v_{n}$ has a convergent subsequence $v_{n_{i}} \rightarrow v$. Now we claim that $v \in P_{V}\left(x_{0}\right)=$ $\cap_{n=1}^{\infty} P_{V}\left(x_{n}\right)$. Let $n$ be fixed but arbitrary. Consider

$$
\begin{aligned}
d\left(x_{n}, v\right) & =d\left(x_{n}, \lim v_{n_{i}}\right) \\
& =\lim _{i} d\left(x_{n}, v_{n_{i}}\right) \\
& \leqslant \lim _{i}\left[d\left(x_{n}, x_{n_{i}}\right)+d\left(x_{n_{i}}, v_{n_{i}}\right)\right] \\
& =\lim _{i}\left[d\left(x_{n}, x_{n_{i}}\right)+d\left(x_{n_{i}}, V\right)\right] \\
& =\lim _{i}\left[d\left(x_{n}, x_{n_{i}}\right)+d\left(x_{n_{i}}, v_{0}\right)\right] \\
& =d\left(x_{n}, x_{0}\right)+d\left(x_{0}, v_{0}\right) \\
& =d\left(x_{n}, v_{0}\right) \\
& =d\left(x_{n}, V\right) .
\end{aligned}
$$

This gives $v \in P_{V}\left(x_{n}\right)$ for all $n$ i.e. $v \in \cap_{n=1}^{\infty} P_{V}\left(x_{n}\right)=P_{V}\left(x_{0}\right)$. Thus $v \in P_{V}\left(x_{0}\right) \subset W$. As $W$ is open, there exists $N$ such that $v_{n} \in W$ for all $n \geqslant N$. Thus $P_{V}\left(x_{n}\right) \subset W$ for all $n \geqslant N$. Therefore for some $\delta>0, P_{V}(x) \subset W$ for all $x$ in $G_{1}\left(v_{0}, x_{0},-\right) \cap B\left(x_{0}, \delta\right)$ and so there exists a neighbourhood $U$ of $x_{0}$ such that $P_{V}(x) \subset W$ for all $x \in G_{1}\left(v_{0}, x_{0},-\right)$. Therefore $P_{V}$ is ORU-continuous at $x_{0}$ and hence $P_{V}$ is ORU-continuous. 
Corollary 5.1. If $V$ is a Chebyshev sun in an externally convex Mspace $(X, d)$ then $P_{V}$ is ORU-continuous.

Note 5.2. For normed linear spaces, Theorem 5.1 is given in [4].

\section{Inner Radial Upper (IRU) Continuity}

A second generalization of usc is:

Let $V$ be a non-empty subset of a convex metric space $(X, d)$ and $x_{0} \in X$. We say that $P_{V}$ is IRU-continuous at $x_{0}$ if for each $v_{0} \in P_{V}\left(x_{0}\right)$ and each open set $W \supset P_{V}\left(x_{0}\right)$ there exists a neighborhood $U$ of $x_{0}$ such that $P_{V}(x) \subset W$ for all $x \in U \cap W\left(v_{0}, x_{0}, \lambda\right)$. $P_{V}$ is called IRUcontinuous if it is IRU-continuous at each point.

Concerning IRU-continuity in convex metric space we have:

Proposition 6.1. Let $V \subset X$ and $x_{0} \in X$. Consider the following statements:

1. $P_{V}$ is IRU-continuous at $x_{0}$.

2. For each $v_{0} \in P_{V}\left(x_{0}\right)$ and each $\epsilon>0$ there exists a $\delta>0$ such that $\sup d\left(v, P_{V}\left(x_{0}\right)\right)<\epsilon$ for every $x$ in $\left\{W\left(v_{0}, x_{0}, \lambda\right): \lambda \in[0,1]\right\}$ $v \in P_{V}(x)$

with $d\left(x, x_{0}\right)<\delta$.

3. For each $v_{0} \in P_{V}\left(x_{0}\right)$ and each sequence $x_{n}$ in $W\left(v_{0}, x_{0}, \lambda\right)$ with $x_{n} \rightarrow x_{0}, \sup _{v \in P_{V}\left(x_{n}\right)} d\left(v, P_{V}\left(x_{0}\right)\right) \rightarrow 0$.

4. For each $v_{0} \in P_{V}\left(x_{0}\right)$ and each sequence $x_{n}$ in $W\left(v_{0}, x_{0}, \lambda\right)$ with $x_{n} \rightarrow x_{0}$ and each sequence $v_{n}$ with $v_{n} \in P_{V}\left(x_{n}\right), d\left(v_{n}, P_{V}\left(x_{0}\right)\right) \rightarrow$ 0 .

5. For each $v_{0} \in P_{V}\left(x_{0}\right)$ and each sequence $x_{n}$ in $W\left(v_{0}, x_{0}, \lambda\right)$ with $x_{n} \rightarrow x_{0}$ and each sequence $v_{n}$ with $v_{n} \in P_{V}\left(x_{n}\right)$ and $v_{n} \rightarrow v$, $v \in \overline{P_{V}\left(x_{0}\right)}$.

Then $(1) \Rightarrow(2) \Leftrightarrow(3) \Leftrightarrow(4) \Rightarrow(5)$. Moreover if $P_{V}\left(x_{0}\right)$ is compact, $(4) \Rightarrow(1)$ and the first four statements are equivalent. If $V$ is compact then $(5) \Rightarrow(1)$ and hence all the five statements are equivalent. 
Proof. (1) $\Rightarrow(2)$ Choose $v_{0} \in P_{V}\left(x_{0}\right)$ and $W=\bigcup\left\{B\left(v, \frac{\epsilon}{2}\right): v \in P_{V}\left(x_{0}\right)\right\} \supset$ $P_{V}\left(x_{0}\right)$. By the IRU- continuity, there exists a neighbourhood $B\left(x_{0}, \delta\right)$ of $x_{0}$ such that $P_{V}(x) \subset W$ for every $x$ in $B\left(x_{0}, \delta\right) \cap\left\{W\left(v_{0}, x_{0}, \lambda\right): \lambda \in\right.$ $[0,1]\}$.

Let $x \in B\left(x_{0}, \delta\right) \cap\left\{W\left(v_{0}, x_{0}, \lambda\right): \lambda \in[0,1]\right\}$ and $v \in P_{V}(x)$ then there exists $v^{\prime} \in P_{V}\left(x_{0}\right)$ such that $d(v, v \prime)<\frac{\epsilon}{2}$ and so $d\left(v, P_{V}\left(x_{0}\right)\right)<\frac{\epsilon}{2}$ as $d\left(v, P_{V}\left(x_{0}\right)\right)=\inf _{w \in P_{V}\left(x_{0}\right)} d(v, w)$. It follows that $\sup _{v \in P_{V}(x)} d\left(v, P_{V}\left(x_{0}\right)\right) \leqslant$ $\frac{\epsilon}{2}<\epsilon$.

$(2) \Rightarrow(3)$ Let $v_{0} \in P_{V}\left(x_{0}\right)$ and $x_{n}$ be a sequence in $W\left(v_{0}, x_{0}, \lambda\right)$ with $x_{n} \rightarrow x_{0}$. Therefore, for $\delta>0$ there exists a positive integer $N$ such that $d\left(x_{n}, x_{0}\right)<\delta$ for all $n \geqslant N$. If $n \geqslant N, x_{n} \in B\left(x_{0}, \delta\right) \cap W\left(v_{0}, x_{0}, \lambda\right)$ and so by the hypothesis, $\sup _{v \in P_{V}\left(x_{n}\right)} d\left(v, P_{V}\left(x_{0}\right)\right)<\epsilon$ for all $n \geqslant N$, which implies $\sup _{v \in P_{V}\left(x_{n}\right)} d\left(v, P_{V}\left(x_{0}\right)\right) \rightarrow 0$.

$(3) \Rightarrow(2)$ Suppose (2) does not hold i.e. there exists $v_{0} \in P_{V}\left(x_{0}\right)$ and $\epsilon>0$ such that for every $\delta>0, \sup _{v \in P_{V}(x)} d\left(v, P_{V}\left(x_{0}\right)\right) \geqslant \epsilon$ for all $x$ in $\left\{W\left(v_{0}, x_{0}, \lambda\right): \lambda \in[0,1]\right\}$ with $d\left(x, x_{0}\right)<\delta$. Let $x_{n}$ be a sequence in $\left\{W\left(v_{0}, x_{0}, \lambda\right): \lambda \in[0,1]\right\}$ with $x_{n} \rightarrow x_{0}$. Then $\sup _{v \in P_{V}\left(x_{n}\right)} d\left(v, P_{V}\left(x_{0}\right)\right) \geqslant \epsilon$ for all $n$ and so $\sup _{v \in P_{V}\left(x_{n}\right)} d\left(v, P_{V}\left(x_{0}\right)\right)$ does not converge to 0 , a contradiction.

$(3) \Rightarrow(4)$ Let $v_{0} \in P_{V}\left(x_{0}\right)$ and $x_{n}$ be a sequence in $W\left(v_{0}, x_{0}, \lambda\right)$ with $x_{n} \rightarrow x_{0}$ and $v_{n}$ be a sequence in $P_{V}\left(x_{n}\right)$.

By hypothesis, $\sup _{v \in P_{V}\left(x_{n}\right)} d\left(v, P_{V}\left(x_{0}\right)\right) \rightarrow 0$ and so $d\left(v_{n}, P_{V}\left(x_{0}\right)\right) \rightarrow 0$.

$(4) \Rightarrow(3)$ Suppose (3) does not hold i.e. for some $v_{0} \in P_{V}\left(x_{0}\right)$ there exists a sequence $x_{n}$ in $W\left(v_{0}, x_{0}, \lambda\right)$ with $x_{n} \rightarrow x_{0}$ such that $\sup d\left(v, P_{V}\left(x_{0}\right)\right)$ does not converge to 0 . Let $v_{n}$ be a sequence in $v \in P_{V}\left(x_{n}\right)$

$P_{V}\left(x_{n}\right)$. So we have $\sup _{v_{n} \in P_{V}\left(x_{n}\right)} d\left(v_{n}, P_{V}\left(x_{0}\right)\right)$ does not converge to 0 . This implies $d\left(v_{n}, P_{V}\left(x_{0}\right)\right)$ does not converge to 0 for some $v_{n} \in P_{V}\left(x_{n}\right)$, a contradiction.

$(4) \Rightarrow(5)$ Let $v_{0} \in P_{V}\left(x_{0}\right), x_{n}$ be a sequence in $W\left(v_{0}, x_{0}, \lambda\right)$ with $x_{n} \rightarrow x_{0}$ and $v_{n}$ be a sequence in $P_{V}\left(x_{n}\right)$ with $v_{n} \rightarrow v$. By hypothesis, $d\left(v_{n}, P_{V}\left(x_{0}\right)\right) \rightarrow 0$ i.e. $\lim d\left(v_{n}, P_{V}\left(x_{0}\right)\right)=0$. This implies 
$d\left(v, P_{V}\left(x_{0}\right)\right)=0 \Rightarrow v \in \overline{P_{V}\left(x_{0}\right)}$.

Suppose $P_{V}\left(x_{0}\right)$ is compact. To prove $(4) \Rightarrow(1)$.

Suppose (1) does not hold i.e. there exists $v_{0} \in P_{V}\left(x_{0}\right)$ and some open set $W \supset P_{V}\left(x_{0}\right)$ such that for every $n$ there is an $x_{n}$ in $\left\{W\left(v_{0}, x_{0}, \lambda\right)\right.$ : $\left.1-\frac{1}{n}<\lambda \leqslant 1\right\}$ such that $P_{V}\left(x_{n}\right) \cap W^{c} \neq \emptyset$. Choose $v_{n} \in P_{V}\left(x_{n}\right) \cap W^{c}$. Then $x_{n} \rightarrow x_{0}$ and so $d\left(v_{n}, P_{V}\left(x_{0}\right)\right) \rightarrow 0$. Choose $y_{n} \in P_{V}\left(x_{0}\right)$ such that $d\left(v_{n}, y_{n}\right) \rightarrow 0$. As $P_{V}\left(x_{0}\right)$ is compact, there exists a subsequence $y_{n_{i}} \rightarrow y_{0} \in P_{V}\left(x_{0}\right)$. Consider

$$
d\left(v_{n_{i}}, y_{0}\right) \leqslant d\left(v_{n_{i}}, y_{n_{i}}\right)+d\left(y_{n_{i}}, y_{0}\right) \rightarrow 0
$$

This implies $v_{n_{i}} \rightarrow y_{0}$. Since $y_{0} \in P_{V}\left(x_{0}\right) \subset W, y_{0} \in W$ and $W$ is open, $v_{n} \in W$ for large $n$, a contradiction.

Suppose $V$ is compact. To prove $(5) \Rightarrow(1)$. We show that $(5) \Rightarrow$ (4) $\Rightarrow(1)$.

Let $v_{0} \in P_{V}\left(x_{0}\right)$ and $x_{n}$ be a sequence in $\left\{W\left(v_{0}, x_{0}, \lambda\right): \lambda \in[0,1]\right\}$ with $x_{n} \rightarrow x_{0}$ and $v_{n}$ be a sequence with $v_{n} \in P_{V}\left(x_{n}\right)$. To prove $d\left(v_{n}, P_{V}\left(x_{0}\right)\right) \rightarrow 0$. Since $P_{V}\left(x_{n}\right)$ is a closed subset of the compact set $V$, it is compact and so $v_{n}$ has a subsequence $v_{n_{i}} \rightarrow v$. By hypothesis, $v \in \overline{P_{V}\left(x_{0}\right)}$. Consider

$$
d\left(v_{n_{i}}, P_{V}\left(x_{0}\right)\right) \leqslant d\left(v_{n_{i}}, v\right)+d\left(v, P_{V}\left(x_{0}\right)\right) \rightarrow 0
$$

i.e. (4) is true. Since $V$ is compact, $P_{V}\left(x_{0}\right)$ is compact and so (4) $\Rightarrow(1)$. Hence $(5) \Rightarrow(1)$.

For Chebyshev sets, we have

Theorem 6.1. If $V$ is Chebyshev set in a convex metric space $(X, d)$ then $P_{V}$ is IRU continuous.

Proof. Let $x_{0} \in X$ be arbitrary and $v_{0} \in P_{V}\left(x_{0}\right)$. Let $G$ be an open set with $G \supset P_{V}\left(x_{0}\right)=\left\{v_{0}\right\}$. Let $x \in\left\{W\left(x_{0}, v_{0}, \lambda\right): \lambda \in[0,1]\right\}$ then $v_{0} \in P_{V}(x)[9]$. As $V$ is Chebyshev, $P_{V}(x)=\left\{v_{0}\right\}$. Let $U$ be any neighborhood of $x_{0}$ then for all $x \in U \cap\left\{W\left(x_{0}, v_{0}, \lambda\right): \lambda \in[0,1]\right\}$, $P_{V}(x)=\left\{v_{0}\right\} \subset G$. Hence $P_{V}$ is IRU-continuous. 


\section{$7 \quad$ IRL and ORU Continuity}

In this section, we discuss the structure of the set having both IRL and ORU continuous metric projection.

A subset $V$ of a metric space $(X, d)$ is said to be boundedly connected if $V \cap B(x, r)$ is connected for every $x \in X$ and $r>0$.

Brosowski and Deutsch [4] proved that for Banach spaces, a set having both IRL and ORU-continuous metric projection is boundedly connected and have a connected valued metric projection. We now extend this result to externally convex M-spaces.

Theorem 7.1. Let $V$ be a proximinal subset of an externally convex $M$-space $(X, d)$ such that $P_{V}$ is both IRU and ORU-continuous then $V$ is boundedly connected and $P_{V}(x)$ is connected for each $x$ in $X$.

Proof. Suppose $V$ is not boundedly connected. Then there exists some $x_{0} \in X$ and a positive $r>d\left(x_{0}, V\right)$ such that $V \cap B\left(x_{0}, r\right)$ is not connected $\left(d\left(x_{0}, V\right) \geqslant r \Rightarrow V \cap B\left(x_{0}, r\right)=\emptyset\right.$ and so there is nothing to prove). Let $V \cap B\left(x_{0}, r\right)=E \cup F$, where $E$ and $F$ are nonempty disjoint sets which are open in $V$. We claim that $P_{V}\left(x_{0}\right) \subseteq E \cup F$.

Let $v_{0} \in P_{V}\left(x_{0}\right)$ i.e. $v_{0} \in V$ and $d\left(x_{0}, v_{0}\right)=d\left(x_{0}, V\right)<r$. Therefore $v_{0} \in V \cap B\left(x_{0}, r\right)=E \cup F$ and so $P_{V}\left(x_{0}\right) \subseteq E \cup F$. We may assume $P_{V}\left(x_{0}\right) \cap E \neq \emptyset$ as $P_{V}\left(x_{0}\right) \subseteq E \cup F$, where $E$ and $F$ are non-empty disjoint sets open in $V$ implies $P_{V}\left(x_{0}\right)$ will have some points common with $E$ or $F$. Let $y \in F$ then there is a $\lambda_{0} \in(0,1)$ such that for every $\lambda \in\left[\lambda_{0}, 1\right], P_{V}\left(W\left(y, x_{0}, \lambda\right)\right) \subseteq F$. Let

$$
\beta=\inf \left\{\lambda \in[0,1]: P_{V}\left(W\left(y, x_{0}, \lambda\right)\right) \subseteq F\right\} .
$$

We first note that $P_{V}\left(W\left(y, x_{0}, \beta\right)\right) \subseteq F$. For if not, then $P_{V}\left(W\left(y, x_{0}, \beta\right)\right) \cap$ $E \neq \emptyset$ (we have $P_{V}\left(W\left(y, x_{0}, \lambda\right)\right) \subseteq E \cup F$ for all $\lambda \in[0,1]$ as $v_{0} \in$ $P_{V}\left(W\left(y, x_{0}, \lambda\right)\right)$. This implies $d\left(W\left(y, x_{0}, \lambda\right), v_{0}\right) \leqslant d\left(W\left(y, x_{0}, \lambda\right), v\right)$ for all $v \in V$ and therefore $d\left(W\left(y, x_{0}, \lambda\right), v_{0}\right) \leqslant d\left(W\left(y, x_{0}, \lambda\right), y\right)=$ $(1-\lambda) d\left(y, x_{0}\right)<(1-\lambda) r$ as $y \in F \subseteq E \cup F=V \cap B\left(x_{0}, r\right)$.Consider $d\left(x_{0}, v_{0}\right) \leqslant d\left(x_{0}, W\left(y, x_{0}, \lambda\right)\right)+d\left(W\left(y, x_{0}, \lambda\right), v_{0}\right)<\lambda r+(1-\lambda) r=r$ and so $\left.v_{0} \in V \cap B\left(x_{0}, r\right)=E \cup F\right)$. Choose $v_{0} \in P_{V}\left(W\left(y, x_{0}, \beta\right)\right) \cap E$. For any sequence $x_{n} \in G\left[W\left(y, x_{0}, \beta\right), y\right]$ such that $x_{n} \rightarrow W\left(y, x_{0}, \beta\right)$, 
there exists (by IRL-continuity) $v_{n} \in P_{V}\left(x_{n}\right) \subseteq F\left(x_{n} \in G\left[W\left(y, x_{0}, \beta\right), y\right] \Rightarrow\right.$ $P_{V}\left(x_{n}\right) \subseteq F$ such that $v_{n} \rightarrow v_{0} \in E$. But this is not possible as $E$ is open in $V$ and $v_{n} \in F \backslash E$ for every $n$. Thus $P_{V}\left(W\left(y, x_{0}, \beta\right)\right) \subseteq F$.

We now claim that best approximations to $W\left(y, x_{0}, \beta\right)$ lie on the line joining $W\left(y, x_{0}, \beta\right)$ and $y$. Suppose this is not true. Then there exists some $v \in F$ not on the line segment joining $W\left(y, x_{0}, \beta\right)$ and $y$ such that $v$ is a best approximation to $W\left(y, x_{0}, \beta\right)$ i.e.

$$
d\left(W\left(y, x_{0}, \beta\right), v\right) \leqslant d\left(W\left(y, x_{0}, \beta\right), z\right) \text { for all } z \in F .
$$

Since $\beta=\inf \left\{\lambda \in[0,1]: P_{V}\left(W\left(y, x_{0}, \lambda\right)\right) \subseteq F\right\}$, we can find a sequence $\beta_{n}$ such that $P_{V}\left(W\left(y, x_{0}, \beta_{n}\right)\right) \subseteq F$ and $\beta_{n} \rightarrow \beta$. Then from (7.1), we get $d\left(W\left(y, x_{0}, \beta\right), v\right)<d\left(W\left(y, x_{0}, \beta\right), W\left(y, x_{0}, \beta_{n}\right)\right)$ as $\left(W\left(y, x_{0}, \beta_{n}\right)\right.$ lie on the line segment joining $W\left(y, x_{0}, \beta\right)$ and $y$ and so cannot be a best approximation $)=d\left(W\left(y, x_{0}, \beta\right), y\right)-d\left(W\left(y, x_{0}, \beta_{n}\right), y\right)=(1-$ $\beta) d\left(y, x_{0}\right)-\left(1-\beta_{n}\right) d\left(y, x_{0}\right)=\left(\beta_{n}-\beta\right) d\left(y, x_{0}\right) \rightarrow 0$.

So, $d\left(W\left(y, x_{0}, \beta\right), v\right)<0$ as equality is not true in view of definition of $v$, a contradiction. Thus best approximation to $W\left(y, x_{0}, \beta\right)$ lie on the line segment joining $W\left(y, x_{0}, \beta\right)$ and $y$.

Let $W\left(y, x_{0}, \alpha\right) \in P_{V}\left(W\left(y, x_{0}, \beta\right) \subseteq F\right.$ with $\alpha>\beta$.

By the ORU-continuity of $P_{V}$, there exists a neighborhood $B_{k}\left(W\left(y, x_{0}, \beta\right)\right)$ such that

$P_{V}(x) \subseteq F$ for every $x$ in $B_{k}\left(W\left(y, x_{0}, \beta\right)\right) \cap\left\{G_{1}\left(W\left(y, x_{0}, \alpha\right), W\left(y, x_{0}, \beta\right),-\right)\right\}$. Choose $\epsilon<\frac{k}{d\left(x_{0}, y\right)}$. Consider

$$
\begin{aligned}
d\left(W\left(y, x_{0}, \beta\right), W\left(y, x_{0}, \beta-\epsilon\right)\right)= & d\left(x_{0}, W\left(y, x_{0}, \beta\right)\right)- \\
& d\left(x_{0}, W\left(y, x_{0}, \beta-\epsilon\right)\right) \\
= & \beta d\left(x_{0}, y\right)-(\beta-\epsilon) d\left(x_{0}, y\right) \\
= & \epsilon d\left(x_{0}, y\right) \\
< & k .
\end{aligned}
$$

Therefore $W\left(y, x_{0}, \beta-\epsilon\right) \in B_{k}\left(W\left(y, x_{0}, \beta\right)\right)$.

Also $W\left(y, d_{0}, \beta-\epsilon\right) \in\left\{G_{1}\left(W\left(y, x_{0}, \alpha\right), W\left(y, x_{0}, \beta\right),-\right)\right\}$ and so $P_{V}\left(W\left(y, x_{0}, \beta-\epsilon\right)\right) \subseteq F$, which contradicts the definition of $\beta$ and hence $V$ is boundedly connected. The proof that $P_{V}(x)$ is connected for each $x$ is virtually the same. 
Corollary 7.1. Let $V$ be a Chebyshev set in an externally convex Mspace satisfying Property (I) and $P_{V}$ is ORU-continuous. Then $V$ is boundedly connected.

Proof. Since $V$ is Chebyshev, $P_{V}$ is IRL-continuous (Corollary 4.1) and the proof follows.

Corollary 7.2. Let $V$ be a Chebyshev sun in an externally convex M-space satisfying Property (I) then $V$ is boundedly connected.

Proof. Since $V$ is Chebyshev, $P_{V}$ is IRL-continuous (Corollary 4.1). Also, $P_{V}(x)$ is compact as $V$ is Chebyshev. Since $V$ is a sun and $P_{V}(x)$ is compact, $P_{V}$ is ORU-continuous (Theorem 5.1). Hence $V$ is boundedly connected

\section{References}

[1] D. Amir and F. Deutsch, Suns, moons and quasi-polyhedra, J. Approx. Theory, 6, (1972), 176-201.

[2] Dietrich Braess, Nonlinear Approximation Theory, Springer-Verlag, Berlin, 1986.

[3] Bruno Brosowski and Frank Deutsch, Some new continuity concepts for metric projections, Bull. Amer. Math. Soc., 78, (1972), 974-978.

[4] Bruno Brosowski and Frank Deutsch, Radial Continuity of set-valued metric projections, J. Approx. Theory, 11, (1974), 236-253.

[5] N. V. Efimov and S. B. Steckin, Some properties of Chebyshev sets, Dokl. Akad. Nauk SSSR, 118, (1958), 17-19.

[6] Shigeru Itoh, Some fixed point theorems in metric spaces, Fundamenta Mathematicae, 52, (1979), 109-117.

[7] Roshdi Khalil, Best approximation in metric spaces, Proc. Amer. Math. Soc., 103, (1988), 579-586.

[8] Hrushikesh N. Mhaskar and Devidas V. Pai, Fundamentals of Approximation Theory, Narosa Publ. House, New Delhi, 2000.

[9] T. D. Narang, Strict convexity and approximation in metric spaces, Caribb. J. Math. Comput. Sci., 8, (1998), 32-42.

[10] B. B. Panda, Some results on best approximations and farthest points, $\mathrm{Ph} . \mathrm{D}$ Thesis, IIT Kanpur, 1974. 
[11] Dale Rolfsen, Geometric methods in Topological spaces, Topology Conference, Arizona State University, 1967.

[12] W. Takahashi, A convexity in metric spaces and non-expansive mappings I, Kodai Math. Sem. Rep., 22, (1970), 142-149.

[13] Shavetambry Tejpal and T. D. Narang, On local and global best approximation, 45, 2007

T. D. Narang

Department of Mathematics

Guru Nanak Dev University

Amritsar

India

E-mail: tdnarang1948@yahoo.co.in

Shavetambry Tejpal

E-mail: shwetambry@gmail.com

Received: 4.04.2012

Accepted: 14.06.2012 